\title{
The Prediction of Prevalence and Spread of HIV/AIDS using Artificial Neural Network - the Case of Rivers State in the Niger Delta, Nigeria
}

\author{
Barileé Baridam \\ Department of Computer Science \\ University of Port Harcourt \\ Nigeria
}

\author{
Chukwuma Irozuru \\ Department of Computer Science \\ University of Port Harcourt \\ Nigeria
}

\begin{abstract}
HIV/AIDS has gained popularity and sufficient research time in the last two centuries. Research has shown that it is most predominant in people between the ages of 15-50. A lot of government and nongovernment organizations have been actively involved in finding ways to help monitor and curb the spread of the disease. Hitherto, there is no clear relevant predictive service available to HIV/AIDS control and research agencies. In this paper, the artificial neural network (ANN) is used in the prediction of prevalence and spread of HIV/AIDS. Results from a detailed analysis of a sample data used prove the robustness of the method.
\end{abstract}

\section{General Terms}

Neural Network, HIV, Prevalence Prediction.

\section{Keywords}

HIV/AIDS Prediction, Artificial Neural Network.

\section{INTRODUCTION}

Scientific discovery and technological development has led to the invention of computer systems which enable complex calculations and integration with sophisticated databases. However, advancement in technology, especially with the development of software, has resulted in an improvement in real time calculation of vital statistical data with high accuracy, precision and speed. Now, calculations and processing programs can be better handled. Various modern ways of calculating statistical data have been invented worldwide by not just the statistical society but also by various companies and organizations that need to know the true state of the parameters of the task at hand, and have basis for prediction of future states of those parameters. With the advent of latest technology, should we fail to update our system, our organizations would record a loss in effectiveness with time. A computer program with the ability to handle complex calculations at real time will help the Medical Society save thousands of lives by providing updated information on HIV/AIDS in the society that would normally have been ignored or given a random value due to the heavy work load required to acquire desired results to a near accurate value. Till date no specialized software for HIV/AIDS statistics is in use, hence the need for this research. However, there has been concentrated research on the HIV/AIDS epidemic since its emergence in the United States (probably enters Haiti around 1966) in 1970. According to a BBC commentator on February 29, 2012, the disease has been spread for more than a 100 years to date. Because of its devastating effect, a concentrated concern has also emerged due to the spread of the HIV/AIDS. Government and nongovernment organizations are not left out. A situational analysis of the HIV epidemic and policy response in Honduras was conducted by the World Bank [25]. HIV/AIDS is a global crisis, a challenge to human life and dignity with ability to erode social and economic development. It has great influence on stability, life expectancy and economic development. It is a major public health problem with SubSaharan Africa severely affected by the epidemic [12]. HIV has the potential of hindering the realization of the Millennium Development Goals and its spread promotes poverty, and has unleashed immense suffering on different countries and communities worldwide [22], and hence, the need to automatically predict the prevalence and spread of the disease based on some available user-inputs data.

\section{DETERMINATION OF}

\section{PREVALENCE}

By definition, prevalence of a disease in a statistical population is the total number of cases of the risk factor in the population at a given time, or the total number of cases in the population, divided by the number of individuals in the population. It shows the degree of infection of a disease within a time period in a population.

Given the number of people in a population with the disease at a given time as $a$, and the number of people in the same population that are at risk of developing the same disease within a time frame, excluding those presently with the disease, as $b$. Prevalence can be defined as

$$
\text { prevalence }=\frac{a}{a+b}
$$

Prevalence may also be expressed in terms of subgroups of the population based on different denominator data.

Prevalence is better expressed as a percentage. It is calculated by multiplying the ratio of infected people per population by 100. A second way of expressing prevalence is as the number of cases per 100,000 of the population. It is easier to report a result as ' 87 cases per 100,000 people' than to state the prevalence as 0.00087 .

Le and Boen [23] provide further examples of the calculation of prevalence. According to Le and Boen, prevalence is contrasted with incidence which is a measure of new cases. With this in mind prevalence is deduced as the product of incidence and duration.

$$
\text { Prevalence }=\text { Incidence } * \text { Duration }
$$


$\mathrm{X}_{1} \quad \mathrm{~W}_{1}$

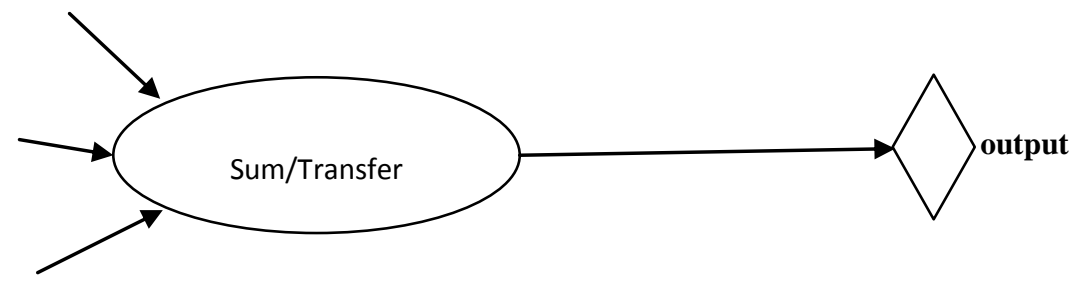

$X_{n} \quad W_{n} \quad$ Processing element

Figure 1. An artificial neuron

Generally, to calculate prevalence with whichever method, the following values must first be determined:

1. The number of cases of the given condition during a specified time period (for HIV/AIDS, usually one year).

2. The total size of the population in which the condition or health indicator occurs (those infected inclusive).

With this two values, prevalence can then be calculated by dividing the number of cases of the condition during the specified time period by the total size of the population in which the condition occurs. As stated earlier, the result is best expressed as a percentage.

According to Le and Boen, the incidence of a disease is another epidemiological measure and it measures the rate of occurrence of new cases of a disease or condition. Incidence is the number of new cases of a condition in a specified time period (usually a year) divided by the total population under consideration who were initially disease free. For example, if a population initially contains 1,000 non-diseased persons and 13 of them develop a condition over a year of observation, the incidence proportion is 13 cases per 1,000 i.e. $1.3 \%$.

It is important to state at this point that the actual report on incidence and prevalence estimates is generally not adequate since the data is only based on number of reported cases [24]. The prediction done in this paper is, therefore, not $100 \%$ free from error since the calculation is only based on available data which may not be an entire capture of the reality.

The UNAIDS have developed software packages and methods that researchers can use to calculate prevalence in a population [25] - [24]. From materials available from UNAIDS/WHO, 'Uniting the world against AIDS' [24], countries with very little available prevalence data, a point prevalence estimate and projection is made using spreadsheet models available through the Workbook Method [24], [25]. The resulting point prevalence estimates for several years are entered into the estimation and projection package (EPP) [22] to find the best fitting curve that describes the evolution of adult HIV prevalence over time. Incidence is then calculated from the prevalence over time [24].

\section{ARTIFICIAL NEURAL NETWORK}

The basic unit of a neural network is the artificial neuron [12] depicted in Fig. 1.

In the figure, $X_{1}, X_{2}, \ldots X_{\mathrm{n}}$ are the inputs to the network, while $W_{l}, W_{2}, \ldots W_{n}$ are the respective connection weights. Each input is multiplied by the appropriate connection weight, the products are summed and fed through a transfer function which generates an output [13]. These neurons are connected in a distinct layer topology to get a network. Some neurons are in the input layer, some others in the middle, that is, the hidden layers, while the rest are in the output layer. A neuron has two modes of operation, the training and the use mode. In the training mode, the neuron is taught to fire (or not) for a particular input pattern while in the use mode, when a taught pattern is detected at the input, its associated output becomes the current output.

A basic network has a feed-forward structure which means that signals flow from the input, forward through hidden units to the output units. A single layer feed-forward network usually consists of inputs, a single layer, the output, and maybe, a bias. Most multilayered networks consist of three or four layers made up of one input layer and one output layer with one or more hidden layers depending on the problem [26]. The input serves to introduce the values of input variables. The hidden and output layers are all connected to the units of the preceding layer. Generally when artificial neural networks are executed, the input variables are placed in the input neurons and the hidden and output neuron are progressively executed. Each of them calculates its own activation value by taking the weighted sum of the output units in the preceding layer. The activation value is then passed through an activation function to produce the output of the neuron. The outputs of the output layer acts as the output of the entire network. Figure 2 illustrates a typical structure of artificial networks.

\subsection{Learning in Neural Networks}

Neural networks have to be trained to associate a given outcome with a given set of inputs [12]. While learning different inputs, the weight values are changed dynamically until their values are balanced so each input will lead to the desired output [27]. The parameters (weights) of neural network model, as in any statistical model, need to be estimated before the network can be used for prediction purposes [26]. Usually the training of a network leads to a matrix that holds the weight values between the neurons and once it has been trained correctly, it can find a desired output 
using these values. Since there are certain errors in the learning process, the generated output is an approximation of the perfect output.

About the commonest learning algorithm employed in multilayered networks is the back propagation algorithm, which is a special form of the Delta learning rule [26]. The algorithm uses the computed output error to change the weight values in the backward direction like the name implies. The net error is first gotten by using the phases in the forward propagation algorithm. During the forward propagation, the output of each neuron in the hidden layer is passed through the sigmoid activation function:

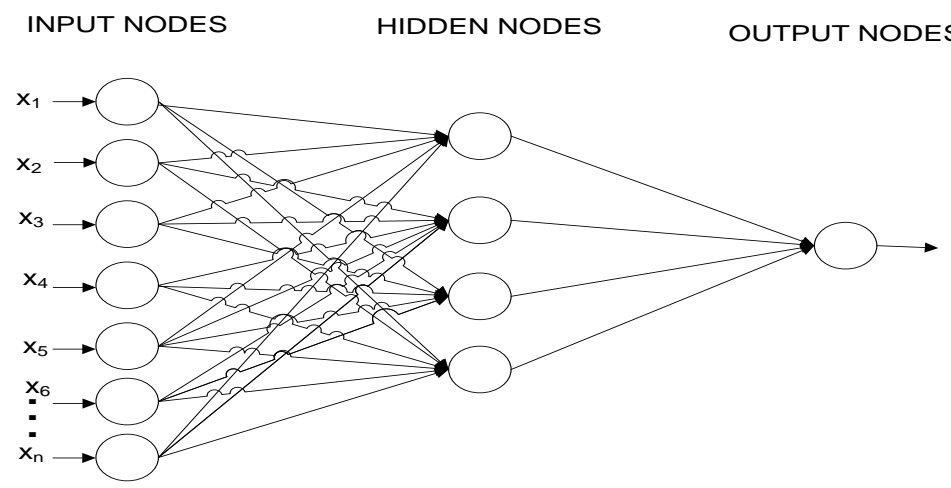

Figure 2. The three layers structure of an Artificial Neural Network

$$
f(x)=\frac{1}{1+\exp (-x)}
$$

A neuron fires, i.e., contributes to the output of the network, only if the value of the function exceeds the threshold value for the neuron.

Activation functions for the hidden units are needed to introduce non-linearity into the networks. Without nonlinearity, hidden units would not make nets more powerful than just plain perceptrons (which do not have any hidden units, just input and output units). The reason is that a composition of linear functions is again a linear function. However, it is the non-linearity (i.e., the capability to represent nonlinear functions) that makes multi-layer networks so powerful. The sigmoid function is the most commonly used activation function in neural networks [28]. The sigmoid output varies continuously but not linearly as the input changes and has been found to bear a great resemblance to the behavior of real neurons [20], [19].

The neural network element computes a linear combination of its input signals, and applies the bounded sigmoid function to the result; this ensures that the output is kept within desired range, that is, 0 - 1 . Inputs entering a neuron not only get multiplied by weights, they also get multiplied by the neurons characteristic equation, or transfer function. The sigmoid function is a typical neuronal non-linear transfer function that helps make outputs reachable. The non-linearity is significant for a further reason. If the transfer function were linear, each of the neuronal inputs would get multiplied by the same proportion during training. This could cause the entire system to "drift" during training runs. That is, the system may lose outputs it has already tracked while attempting to track new outputs. A non-linearity in the system helps to isolate specific input pathways [20]. The behaviour of the sigmoid function is shown in Figure 3.

The training algorithm works as follows:
Step 1: Perform the forward propagation phase for an input pattern and calculate the output error.

Step 2: Change all the weight values in the weight matrix using the formula

Weight (old) + value of change in weights $(c w)$, where

$c w=$ learning rate $*$ output error $*$ output (neuron $i) *$ output(neuron $i+1) *[1-$ output (neuron $i+1)]$

Step 3: Go to step 1

Step 4: The algorithm is repeated until the maximum number of training sessions have been executed.

The first input pattern is propagated through the network using randomly selected initial weights. The same procedure is carried out for the next input pattern but now with the changed weight values. After the feed-forward and back ward propagation of the second to the last input patterns, one learning step is completed and the grand error is calculated by summing the square of the output errors of all the patterns. This procedure is repeated until the error gets to zero or an approximation of zero.

The training process can be seen as an optimization problem, where we wish to minimize the mean square error of the entire set of training data. Over the training step, the network converges to an optimal set of weights, which gives a network that can handle any arbitrary pattern that belongs to the same class as the training set. So, the training normally starts with a set of arbitrary values in the range -1 to +1 and can even be set using a random number generator. The back-propagation training step ensures that each of the weights converge to its optimal value for the network [21].

Besides so many applications of artificial neural network, it is interesting to know that the field of medical technology has not been left out. ANN been employed in the prediction of rate of death in patients with hepatitis-c virus infection [12], the prediction of coronary artery disease [22], prediction of 
diabetes [23], general medical disease predictions[24], and Hepatitis-C diagnosis [25].

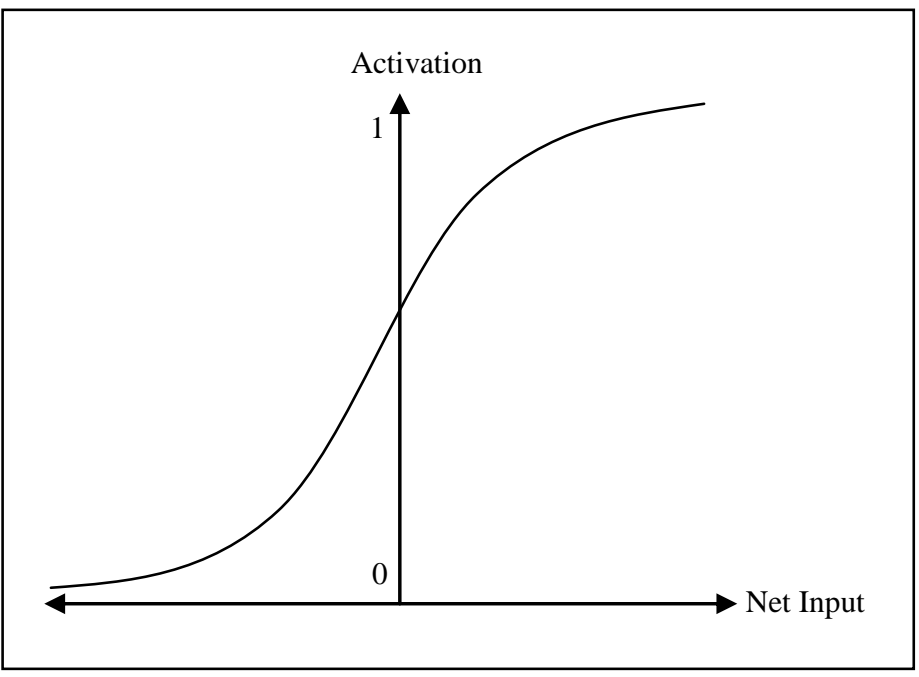

Figure 3. Sigmoid Activation Function

\section{THE DESIGN OF THE NEURAL NETWORK MODEL}

The main plan of this software is to create a simple user interface that is connected to a database and capable of handling hundreds of thousands of fields through a weighted neuron for the main purpose of calculating HIV/AIDS prevalence and storing of related data. The database is equipped with the capacity of accepting queries for various results simultaneously with little or no user input, and the ability accept queries for specific and general results. Query results are relayed through output neurons and are displayed when the software loads and after button-clicks depending on the information the user desires. Common and highly relevant information are automatically loaded when the reports window loads to reduce the need for user input.

Besides, the software also has the ability to rapidly and securely commit information related to HIV/AIDS in a database. Relevant information from the input neurons is displayed in a grid for a general view. Future prevalence is also calculated when the user sends an input signal in value corresponding to the number of years ahead that he/she wishes to know the projected future fate of prevalence. Graphical representations including pie charts, bar charts, graphs, doughnut charts etc. representing various classifications of patient data are also plotted to give a graphical representation of results. Input variables for the network design are: Age, Sex, Marital Status, Pregnancy Status, Mode of Infection (MOI), Religion, and Level of Education. Data were collected under strict adherence to the medical profession ethics. Professionals were consulted Laboratory Technicians, Nurses, and Medical Doctors. Input from these professionals gave rise to the expert system to answer their questions as follows: the presentation of prevalence output (results) based on age groups in a graphical form; prevalence result among pregnant women; and the automatic generation of prevalence predictions.

Neural network model employs a training process i.e., the process of determining the weights. During the training sessions, several weights are introduced and the behaviour of the network in response to the weight adjustments determines what value will be used further. What determines the parameter (weight) used in the prediction of prevalence of HIV/AIDS is the awareness factor. It is expected that the percentage of awareness, to a large extend, influences the rate of infection of the disease. The training weights introduced for the hidden layer were: 1 (Excellent), 2 (Good), 3 (Average), 4 (Fair), and 5 (Poor). The predictor variables are multiplied by a constant input weight, which may be referred to as the bias, based on the level of education in the area under investigation. The value is low or high depending on whether there is a high or low level of education respectively. As could be seen from Figure 6, the input layer has one neuron for each predictor variable.

The input layer is a collection of a vector of prediction variable values that are distributed to each of the hidden layer neurons. At this layer, the bias is added to the sum going into the neuron.

Table 1. Summary of Input and Output Variables

\begin{tabular}{|l|l|}
\hline $\begin{array}{l}\text { Demographic } \\
\text { Characteristics }\end{array}$ & \multicolumn{2}{|l|}{ Specifications } \\
\hline Sex & $0 \& 1$ \\
\hline Marital Status & $0 \& 1$ \\
\hline Age & $0-99$ \\
\hline Mode of Infection (MOI) & $0-5$ \\
\hline Educational level & $\begin{array}{l}1 \text { (KG/Nursery) } \\
2 \text { (Primary) } \\
3 \text { (Secondary) } \\
4 \text { (Tertiary) }\end{array}$ \\
\hline
\end{tabular}




\begin{tabular}{|c|c|c|}
\hline Pregnancy Status & $\mid \begin{array}{l}0 \text { (Not Pregnant) } \\
1 \text { (Pregnant) }\end{array}$ & \\
\hline Religion & $0-3$ & \\
\hline $\begin{array}{l}\text { HIV Prevalence } \\
\text { Prediction }\end{array}$ & $\begin{array}{l}\% \text { Prevalence per population } \\
\% \text { Prevalence per sex }\end{array}$ & Output \\
\hline
\end{tabular}

\section{EXPERIMENTAL RESULTS}

A sample data from the University of Port Harcourt Teaching Hospital (UPTH) was collected and imputed through the neurons. This sample data was limited to only people that have been tested during the period of January 1, 2011 to June 30, 2011. Out of the 2005 estimated population of Rivers State i.e., 6,689,087[26], a total of 800 records of tested people were collected. This figure is a fraction of all tested people that may be available in other health centers across the state. Owing to the fact that the total number of patients that had visited the hospital could just be an average of the entire population, for a more realistic result, certain assumptions have to be made as follows:

- It is assumed that the total number of population has been tested.

- It is assumed that if 800 people are tested in UPTH, at least 83,614 people would have been tested throughout the state.

- It is also assumed that the study is run for a period of 12 months. This is necessary to ensure a sizeable number of patients are covered based on the population of the state under study.

- It is assumed that there were no errors during data collection.

- No other geographic region is in consideration other than the community under investigation.

Some of the information collected from the sample data are presented in Table 2.

Table 2. Information related to data collected

\begin{tabular}{|l|l|}
\hline Age range of sample data & $0-99$ \\
\hline Duration of study & 6 months \\
\hline Total number of sample data & 800 \\
\hline $\begin{array}{l}\text { Total number of HIV positive } \\
\text { people }\end{array}$ & 51 \\
\hline $\begin{array}{l}\text { Total number of HIV } \\
\text { negative people }\end{array}$ & 749 \\
\hline $\begin{array}{l}\text { Total number of HIV positive } \\
\text { male }\end{array}$ & 21 \\
\hline $\begin{array}{l}\text { Total number of HIV positive } \\
\text { female }\end{array}$ & 30 \\
\hline $\begin{array}{l}\text { Total number of HIV positive } \\
\text { in urban areas }\end{array}$ & 25 \\
\hline
\end{tabular}

The prediction of prevalence on a future population is determined based on an increase in population. To obtain the prevalence the following equation is introduced to determine an equivalent population increase using the sample data.

Equivalent population increase in sample data,

$$
P^{\uparrow}=s\left(\frac{c}{a}\right)
$$

where $s=$ sample population, $c=$ yearly increase in real population and $a=$ real population

\subsection{Interpretation of Results}

The prediction of prevalence is obtained from the network. The result from the percentage prevalence assists in the determination of the prevalence value (percentage of population infected), percentage of population infected by sex, percentage of unmarried women affected in population, etc. as presented in Table 3 .

The total HIV/AIDS prevalence presented based on the data collected was $6.5 \%$. This value corresponds closely to the report of George Onah, [27] where prevalence rate of Rivers State is given as $7.4 \%$. We can conclusively say that an approximate of one (1) person in sixteen (16) people is HIV positive.

From the data collected from UPTH, 7 women where pregnant and living with HIV. This gives a prevalence of $0.00875 \%$ or $8.75(9)$ per 1000 and when compared to the 2011 report of Rivers State by George Onah, we find that he reports that 5,162 women were HIV positive giving a prevalence of $0.001 \%$ or 1 per 1000 . This results show a drastic increase in the number of women that are pregnant and HIV positive. This could mean that more women are aware of the many ways of preventing mother-to-child transmission and are confident to get pregnant despite their status. Other statistical information regarding knowledge base of pregnant women could help us confirm this assumption. Ugboma et al, [28] shows that $79.9 \%$ of people in Rivers State are knowledgeable on HIV/AIDS and of this $79.9 \%, 80.5 \%$ are women. This shows that more women are aware of options available to them when HIV positive. It is further confirmed by results that show that only one (1) in a thousand babies that are born would be HIV positive according to the prevalence data. 
Table 3. Present Prevalence based on 2005 population

\begin{tabular}{|l|c|}
\hline Categories & Percentage Prevalence \\
\hline Pregnant teenagers with HIV & $0.25 \%$ \\
\hline Female teenagers with HIV & $0.5 \%$ \\
\hline Pregnant women with HIV & $0.09 \%$ \\
\hline Nursing mothers with HIV & $0.09 \%$ \\
\hline Total female with HIV & $4.3 \%$ \\
\hline Total male with HIV & $2.3 \%$ \\
\hline Total teenagers with HIV & $0.63 \%$ \\
\hline Infant cases (below 1yr) & $0.13 \%$ \\
\hline HIV carriers in urban areas & $3.3 \%$ \\
\hline Pediatric infections (0-14yrs) & $0.25 \%$ \\
\hline $\begin{array}{l}\text { Women above 15yrs (Mother to } \\
\text { Child) }\end{array}$ & $4.1 \%$ \\
\hline Total HIV carriers & $6.5 \%$ \\
\hline Population infected through sex & $3 \%$ \\
\hline Unmarried female carriers & $1.625 \%$ \\
\hline
\end{tabular}

There is an almost 50\% distribution of HIV/AIDS cases when considering the place of residence of confirmed HIV positive people in the sample data. 25 out of the 51 recorded HIV patients were from urban areas and 26 from rural areas. This indicates that there should be an almost equal degree of HIV/AIDS campaign in the rural and urban areas. About 3\% of the total population got infected by unprotected sex. This indicates that almost half of the infected population was caused by unprotected sex.

From the base population of $6.689,087$, if the level of awareness and prevention of HIV/AIDS is not intensified, with the current rate of the epidemic, in 50 years from 2011 HIV/AIDS will have a $15 \%$ toll on the Rivers community assuming a yearly population growth of $0.16 \%$. The number of deaths caused by the disease will also increase. Table 4 presents the percentage prevalence in an interval of 5 years until 2061.
Table 4. Percentage HIV/AIDS prevalence over a period of 50 years

\begin{tabular}{|c|c|}
\hline Year & $\begin{array}{c}\text { Estimated } \\
\text { \% Prevalence }\end{array}$ \\
\hline 2016 & 7.3 \\
\hline 2021 & 8.2 \\
\hline 2026 & 9.1 \\
\hline 2031 & 9.9 \\
\hline 2036 & 10.8 \\
\hline 2041 & 11.6 \\
\hline 2046 & 12.5 \\
\hline 2051 & 13.3 \\
\hline 2056 & 14.2 \\
\hline 2061 & 15.0 \\
\hline
\end{tabular}

\section{CONCLUSION}

The artificial neural network is employed in this paper in the prediction of the prevalence of HIV/AIDS over a specific period of time within Rivers State in the Niger Delta of Nigeria. The results obtained show the robustness of the application, and should be a guide to individuals, government and non-government organizations in their fight against the scourge of HIV/AIDS. The study has the potential of being extended to national populations if HIV/AIDS reports for such a nation are available.

\section{REFERENCES}

[1] The World Bank, Building on Evidence: A Situational Analysis of the HIV Epidemic and Policy Response in Honduras, The Global HIV/AIDS Program. Available at: http://siteresources.worldbank.org/INTHIVAIDS/Resour ces/375798-

1103037153392/HondurasSynthesisSummary.pdf

[2] Buvé, T. and Timothy. C, 2011 HIV/AIDS prevalence and behaviour in drug users and pregnant women in Kashgar Prefecture: Case report. Harm Reduction Journal 3:7, 3-7

[3] Mathew, N. 2011 Nigeria Progress Report. Pages 20-25

[4] Le C.T. and Boen J.R. 1995 Health and numbers: basic biostatistical methods. John Wiley, Chichester.

[5] HIV/AIDS Estimates 2008, HIV \& Hepatitis Epidemiology Program, Spring Quarterly Report: April

[6] Ghys, P. D., Walker N., McFarland W, Miller R, Garnett G. P. 2008 Improved data, methods and tools for the 2007 HIV and AIDS estimates and projections Sexually Transmitted Infections, Volume 84, pp i1-i4, doi:10.1136/sti.2008.032573

[7] Ghys, P. D., Walker N., Garnett G. P. 2006: Improving analysis of the size and dynamics of AIDS epidemics Sexually Transmitted Infections, Volume 82, pp iii1-iii2 doi:10.1136/sti.2006.021030 
[8] Ghys, P. D., Brown, T., Grassly N. C., Garnett G, Stanecki K. A., Stover J., Walker N. 2004 The UNAIDS Estimation and Projection Package: a software package to estimate and project national HIV epidemics, Sexually Transmitted Infections, Volume 80 (Suppl I), pp i5-i9. doi: 10.1136/sti.2004.010199

[9] Lippincott Williams and Wilkins 2011: HIV in hiding: methods and data requirements for the estimation of the number of people living with undiagnosed HIV, Working Group on Estimation of HIV Prevalence in Europe, AIDS, Volume 25, pages 1017-1023

[10] Methods and assumptions for estimates, UNAIDS Report, Available at http://www.unaids.org/en/media/unaids/contentassets/do cuments/dataanalysis/Epi_MethodsAssumptionsEstimate s_en.pdf, visited on February 26, 2012

[11] Natalia Ladnaya 2008 Epidemiologic Models Introduction to Work Book, Russian Federal AIDS Centre, ASAP Regional Training on Strategic and Operational Planning in HIV \& AIDS, September 29 October 10, Budapest, Hungary

[12] Owolabi, O. and Baridam, B. B. 2011 Predicting Mortality in Hepatitis-C Patients Using an Artificial Neural Network. Global Journal of Pure and Applied Science, Volume 17, No. 2, Pages 159-164,

[13] Patterson, D, 1996. Artificial Neural Networks. Prentice Hall, Singapore

[14] Fröhlich, J., 1997. Neural networks with java. http://pages.cpsc.ucalgary.ca/ carman/533/progress/prog ress_report.doc

[15] Haykin, S., 1994. Neural Networks: A Comprehensive Foundation.: Macmillan Publishing, New York.

[16] Wilbert Sibanda and Philip Pretorius 2011 Novel Application of Multi-Layer Perceptrons (MLP) Neural Networks to Model HIV in South Africa using Seroprevalence Data from Antenatal Clinics International Journal of Computer Applications (0975 8887) Volume 35, No.5, Pages 26-31, December.

[17] Fausett, L., 1994. Fundamentals of Neural Networks: Architectures, Algorithms, and Applications. PrenticeHall, Inc.

[18] McCullough, P. and J. A. Nelder, 1989. Generalized Linear Models, $2^{\text {nd }}$ ed., London: Chapman \& Hall.
[19] Alexander, I. and H. Morton, 1995. An Introduction to Neural Computing (Second Edition), Thomson Computer Press.

[20] Anderson, J. A., 1995. An Introduction to Neural Networks. Cambridge, MA/Bradford Books.

[21] McClelland, J. L. and D. E. Rumelhart, (Eds.). 1988. Explorations in parallel distributed processing: A handbook of models, programs, and exercises. Cambridge, MA: MIT Press.

[22] M. Cengiz Çolak, Cemil Çolak, Hasan Kocatürk, Seref Sagiroglu, Irfan Barutçu 2008 Predicting coronary artery disease using different artificial neural network models, $C A D$ and Artificial Neural Network, Volume 8, pages 249-254,

[23] Muhammad Akmal Sapon, Khadijah Ismail and Suehazlyn Zainudin 2011. Prediction of Diabetes by using Artificial Neural Network, International Conference on Circuits, System and Simulation IPCSIT vol.7, pp 299-303

[24] Mantzaris,D.H., Anastassopoulos, G.C., Lymberopoulos, D.K. 2008 Medical disease prediction using Artificial Neural Networks, 8th IEEE International Conference on Bioinformatics and Bioengineering, pp.1-6.

[25] Uttreshwar, G.S.; Ghatol, A.A. 2009 Hepatitis B Diagnosis Using Logical Inference and Generalized Regression Neural Networks, IEEE International Advance Computing Conference, pp.1587-1595.

[26] Rivers State, http://en.wikipedia.org/wiki/Rivers_State

[27] George Onah 2011: Rivers' LG records 4,746 HIV/AIDS positive cases, Vanguard News, October 5, , Available at: http://www.vanguardngr.com/2011/10/rivers-lgrecords-4746-hivaids-positive-cases/

[28] H. A. A. Ugboma, M. E. Kooffreh and C. A. Nwauche 2011 Gender differences in student's knowledge of HIV/AIDS in the Niger Delta, Nigeria Journal of Clinical Medicine and Research Vol. 3(1), pp. 20-22 